\title{
COVID-19-related severe MS exacerbation with life-threatening Takotsubo cardiomyopathy in a previously stable patient and interference of MS therapy with long-term immunity against SARS-CoV-2
}

\author{
Brigitte Wildemann ${ }^{1}$. Sven Jarius ${ }^{1} \cdot$ Lorenz H. Lehmann $^{2} \cdot$ Florian André $^{2} \cdot$ Norbert Frey $^{2} \cdot$ Paul Schnitzler $^{3}$. \\ Laura Jäger ${ }^{4} \cdot$ Christoph Gumbinger $^{4} \cdot$ Andrea Viehöver $^{1}$
}

Received: 23 June 2021 / Revised: 26 August 2021 / Accepted: 26 August 2021 / Published online: 7 October 2021

(C) The Author(s) 2021, corrected publication 2022

\section{Dear Sirs,}

Little is known about how infection with SARS-CoV-2 impacts the course and severity of multiple sclerosis (MS). Here, we report on a patient in whom SARS-CoV-2 infection was followed by severe exacerbation of previously stable MS and Takotsubo cardiomyopathy (TTC). Of note, repeated intravenous pulse therapy with high-dose methylprednisolone (IVMP), plasma exchange, and administration of ocrelizumab interfered with the development of a long-lasting immune response against SARS-CoV-2.

Case report. In a 39-year-old woman, relapsing remitting MS was first diagnosed in 2011 and had been stable since 2014 with continuous administration of dimethyl fumarate (DMF). In December 2020, 10 days after the onset of marked general malaise and fatigue, the patient developed nystagmus, dizziness and headache. The next day she experienced tachycardia, followed the day after by coughing and shortness of breath requiring admission to hospital. Chest CT showed multifocal central and peripheral groundglass opacities involving both pulmonary lobes suggesting

Brigitte Wildemann

brigitte.wildemann@med.uni-heidelberg.de

1 Molecular Neuroimmunology Group, Department of Neurology, University of Heidelberg, Im Neuenheimer Feld 400, 69120 Heidelberg, Germany

2 Department of Cardiology, Angiology and Pneumology, University of Heidelberg, Im Neuenheimer Feld 410, 69120 Heidelberg, Germany

3 Department of Infectious Diseases/Virology, University of Heidelberg, Im Neuenheimer Feld 324, 69120 Heidelberg, Germany

4 Department of Neurology, University of Heidelberg, Im Neuenheimer Feld 400, 69120 Heidelberg, Germany
COVID-19 pneumonia. PCR testing of a throat swab was positive for SARS-CoV-2-RNA and negative for influenza virus. Laboratory tests and ECG screening revealed elevated hs-troponinT $(715 \mathrm{pg} / \mathrm{ml}$; normal $<14)$ and intermittent ventricular bigeminy, respectively. Cardiac catheterization showed no coronary artery disease but regional wall motion abnormalities compatible with atypical TTC. Cranial MRI depicted a symptomatic novel lesion in the dorsomedial medulla oblongata (Fig. 1a, d). The patient was diagnosed with COVID-19 pneumonia, TTC and acute MS relapse. She was treated with oxygen, beta-blocking and angiotensin converting enzyme-inhibiting agents, and IVMP $1000 \mathrm{mg}$ daily for 4 days. Four weeks after symptom onset, the patient noticed sudden deterioration of her general condition along with irregular heart rhythm as well as marked dizziness, double vision, and difficulties on swallowing. Cardiac MRI findings and a renewed increase in hs-troponinT serum levels $(163 \mathrm{pg} / \mathrm{ml})$ were again compatible with TTC (supplemental material). On neurologic assessment, she presented with dysarthria, severe dysphagia requiring tube feeding and marked gait instability. Repeat cranial MRI showed several new brain lesions and enlargement of the brainstem lesion, now contrast-enhancing (Fig. 1b, c, e-g). Cell-based testing for serum myelin oligodendrocyte glycoprotein (MOG)$\operatorname{IgG}[3,6,7]$ and aquaporin-4 (AQP4)-IgG [5] was negative. Cerebrospinal fluid analysis disclosed 4 cells/ $\mu 1$, mild blood-CSF barrier dysfunction, and CSF-restricted oligoclonal IgG bands. The antibody indices for measles and varicella zoster viruses were 2.07 and 1.67, respectively, indicating a positive MRZ reaction, as typically seen in MS but not in MOG encephalomyelitis or AQP4-IgG-positive neuromyelitis optica[1, 2, 4]. CSF-PCR for HSV1/2, varicella zoster virus, and SARS-CoV-2 was negative, as was serology for CSF and serum SARS-CoV-2-IgG. IVMP therapy 

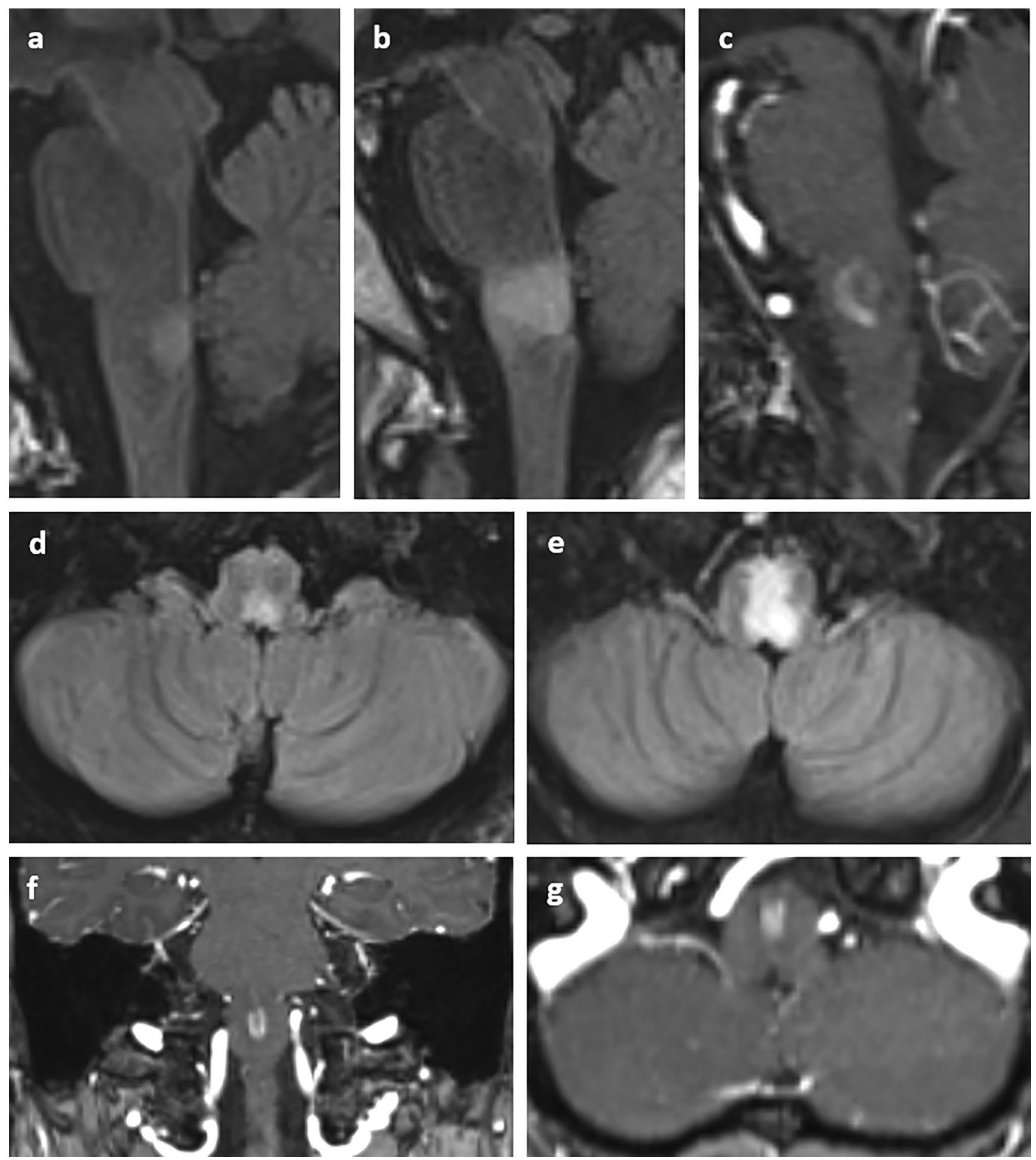

Fig. 1 Cranial MRI 5 days after the onset of neurological symp-

lesion (b), now with gadolinium enhancement (c). d, e Corresponding T2/FLAIR-weighted axial sections at day 5 after symptom onset and 25 days later. Panels $\mathbf{f}, \mathbf{g}$ Gadolinium enhancement of the lesion at enhancement (not shown) in the nucleus tractus solitarii (a) Follow-up MRI 25 days later at the time of neurological deterioration showed enlargement of the same 
$(5 \times 2000 \mathrm{mg}$ daily $)$ and a course of seven plasma exchanges prompted slow improvement of the clinical symptoms. DMF was stopped and replaced by ocrelizumab to control MS disease activity. Lymphocyte counts were normal $(>1 / \mathrm{nl})$ during therapy with DMF (03/2019: 1.5/nl, 04/2020: 1.7/ $\mathrm{nl}, 10 / 2020: 2 / \mathrm{nl}$ ) and at the time of SARS-CoV-2 infection (12/2020: 2.5/nl). Repeat serology for SARS-CoV-2-IgG performed after the last plasma exchange and again 38 days later (i.e., 22 days after ocrelizumab) was negative.

Discussion. This patient developed severe MS activity 10 days after the onset of COVID-19 symptoms. The risk of MS relapses is elevated between 2 weeks before and 5 weeks after the first symptoms of an infection, and viral respiratory tract infections are thought to be the most important triggers[10]. Thus, it appears likely that recent infection with SARS-CoV-2 prompted the pronounced clinical and radiological disease activity in this patient, whose MS had been well controlled for many years on treatment with dimethyl fumarate. This illustrates that SARS-CoV-2 infection might considerably harm MS patients by provoking true MS exacerbation in addition to infection-related pseudorelapses[12]. Of note, the patient had no cardiac comorbidities and denied emotional stress prior to experiencing the cardiac arrhythmia that preceded neurological deterioration. Thus, although TTC - a disorder prompted by a surge in catecholamine levels-has been described as a cardiac complication of COVID-19 [13], altogether it appears more likely that the acute demyelinating lesion in the dorsomedial medulla arising due to breakthrough MS disease activity was responsible for the myocardial dysfunction. The dorsomedial medulla includes the nucleus of the solitary tract, an inhibitory vasomotor center that regulates and dampens sympathetic outflow. Injury to this structure in the context of MS brainstem exacerbations has been reported to cause acute cardiopulmonary events including TTC [8, 9, 11, 14]. In line with previous reports, the course of COVID-19 was moderate overall despite long-term immunotherapy and was also not negatively affected by additional IVMP treatment. In conclusion, this case (i) demonstrates that COVID-19 might be capable of triggering severe and even life-threatening MS exacerbations and suggests that patients with MS should be particularly well protected from SARS-CoV-2 infection; (ii) supports previous evidence suggesting that MS-related brainstem lesions can cause TTC; (iii) arouses concerns regarding the establishment of long-term humoral immunity against COVID-19 in patients treated for MS with plasma exchange, ocrelizumab, steroids and/or other immunotherapies during acute SARS-CoV-2 infection (although a sufficient cellular immune response may still develop, as suggested by the overall favorable outcome from COVID-19 in our patient).
Supplementary Information The online version contains supplementary material available at https://doi.org/10.1007/s00415-021-10779-0.

\section{Declarations}

Conflict of interest BW received grants from the German Ministry of Education and Research, Deutsche Forschungsgemeinschaft, Dietmar Hopp Foundation and Klaus Tschira Foundation, grants and personal fees from Merck, Sanofi Genzyme, Novartis, and personal fees from Alexion, Bayer, Biogen, Teva; none related to this work. SJ reports no disclosures relevant to the article. NF received grants from the German Ministry of Education and Research, Deutsche Forschungsgemeinschaft, and personal fees from AstraZeneca, Bayer, Böhringer Ingelheim, Novartis; none related to this work. LHL has served on the advisory board for Daiichi Sankyio, Senaca, and Servier, as an external expert for AstraZeneca and received speakers' honoraria from Novartis and MSD, none related to this work. AF reports no disclosures relevant to the article. PS reports no disclosures relevant to the article. LJ reports no disclosures relevant to the article. CG reports no disclosures relevant to the article. AV received speaker honoraria from Merck Serono $\mathrm{GmbH}$ and Roche Pharma AG.

Funding Open Access funding enabled and organized by Projekt DEAL

Open Access This article is licensed under a Creative Commons Attribution 4.0 International License, which permits use, sharing, adaptation, distribution and reproduction in any medium or format, as long as you give appropriate credit to the original author(s) and the source, provide a link to the Creative Commons licence, and indicate if changes were made. The images or other third party material in this article are included in the article's Creative Commons licence, unless indicated otherwise in a credit line to the material. If material is not included in the article's Creative Commons licence and your intended use is not permitted by statutory regulation or exceeds the permitted use, you will need to obtain permission directly from the copyright holder. To view a copy of this licence, visit http://creativecommons.org/licenses/by/4.0/.

\section{References}

1. Jarius S, Eichhorn P, Franciotta D, Petereit HF, Akman-Demir G, Wick M, Wildemann B (2017) The MRZ reaction as a highly specific marker of multiple sclerosis: re-evaluation and structured review of the literature. J Neurol 264:453-466

2. Jarius S, Lechner C, Wendel EM, Baumann M, Breu M, Schimmel M, Karenfort M, Marina AD, Merkenschlager A, Thiels C, Blaschek A, Salandin M, Leiz S, Leypoldt F, Pschibul A, Hackenberg A, Hahn A, Syrbe S, Strautmanis J, Hausler M, Krieg P, Eisenkolbl A, Stoffels J, Eckenweiler M, Ayzenberg I, Haas J, Hoftberger R, Kleiter I, Korporal-Kuhnke M, Ringelstein M, Ruprecht K, Siebert N, Schanda K, Aktas O, Paul F, Reindl M, Wildemann B, Rostasy K, in cooperation with the Bsg, the Neuromyelitisoptica Study G (2020) Cerebrospinal fluid findings in patients with myelin oligodendrocyte glycoprotein (MOG) antibodies. Part 2: Results from 108 lumbar punctures in 80 pediatric patients. J Neuroinflam 17:262

3. Jarius S, Paul F, Aktas O, Asgari N, Dale RC, de Seze J, Franciotta D, Fujihara K, Jacob A, Kim HJ, Kleiter I, Kumpfel T, Levy M, Palace J, Ruprecht K, Saiz A, Trebst C, Weinshenker BG, Wildemann B (2018) MOG encephalomyelitis: international recommendations on diagnosis and antibody testing. J Neuroinflam $15: 134$ 
4. Jarius S, Pellkofer H, Siebert N, Korporal-Kuhnke M, Hummert MW, Ringelstein M, Rommer PS, Ayzenberg I, Ruprecht K, Klotz L, Asgari N, Zrzavy T, Hoftberger R, Tobia R, Buttmann M, Fechner K, Schanda K, Weber M, Asseyer S, Haas J, Lechner C, Kleiter I, Aktas O, Trebst C, Rostasy K, Reindl M, Kumpfel T, Paul F, Wildemann B, in cooperation with the NeuromyelitisOptica Study G (2020) Cerebrospinal fluid findings in patients with myelin oligodendrocyte glycoprotein (MOG) antibodies. Part 1: Results from 163 lumbar punctures in 100 adult patients. J Neuroinflam 17:261

5. Jarius S, Probst C, Borowski K, Franciotta D, Wildemann B, Stoecker W, Wandinger KP (2010) Standardized method for the detection of antibodies to aquaporin-4 based on a highly sensitive immunofluorescence assay employing recombinant target antigen. J Neurol Sci 291:52-56

6. Jarius S, Ruprecht K, Kleiter I, Borisow N, Asgari N, Pitarokoili K, Pache F, Stich O, Beume LA, Hummert MW, Ringelstein M, Trebst C, Winkelmann A, Schwarz A, Buttmann M, Zimmermann H, Kuchling J, Franciotta D, Capobianco M, Siebert E, Lukas C, Korporal-Kuhnke M, Haas J, Fechner K, Brandt AU, Schanda K, Aktas O, Paul F, Reindl M, Wildemann B, in cooperation with the NeuromyelitisOptica Study G (2016) MOG-IgG in NMO and related disorders: a multicenter study of 50 patients. Part 2: Epidemiology, clinical presentation, radiological and laboratory features, treatment responses, and long-term outcome. J Neuroinflammation 13:280

7. Jarius S, Ruprecht K, Kleiter I, Borisow N, Asgari N, Pitarokoili K, Pache F, Stich O, Beume LA, Hummert MW, Trebst C, Ringelstein M, Aktas O, Winkelmann A, Buttmann M, Schwarz A, Zimmermann H, Brandt AU, Franciotta D, Capobianco M, Kuchling J, Haas J, Korporal-Kuhnke M, Lillevang ST, Fechner K, Schanda K, Paul F, Wildemann B, Reindl M, in cooperation with the NeuromyelitisOptica Study G (2016) MOG-IgG in NMO and related disorders: a multicenter study of 50 patients. Part 1: Frequency, syndrome specificity, influence of disease activity, long-term course, association with AQP4-IgG, and origin. J Neuroinflammation 13:279

8. Kozu K, Suzuki H, Nishiyama S, Yaoita N, Yamamoto S, Tatebe S, Miura M, Aoki T, Hao K, Matsumoto Y, Sugimura K, Aoki M, Shimokawa H (2016) Multiple sclerosis lesion in the medulla oblongata in a patient with takotsubo cardiomyopathy. Int J Cardiol 222:980-981

9. London F, Rodriguez G, de Azero N, Philippart M, Higny J, Mulquin N (2019) Reverse takotsubo cardiomyopathy triggered by a multiple sclerosis relapse. Acta Neurol Belg 119:295-297

10. Marrodan M, Alessandro L, Farez MF, Correale J (2019) The role of infections in multiple sclerosis. Mult Scler 25:891-901

11. Midaglia L, Juega Marino JM, Sastre-Garriga J, Rovira A, VidalJordana A, Lopez-Perez MA, Marzo-Sola ME, LibradaEscribano F, Montalban X (2016) An uncommon first manifestation of multiple sclerosis: Tako-Tsubo cardiomyopathy. Mult Scler 22:842-846

12. Parrotta E, Kister I, Charvet L, Sammarco C, Saha V, Charlson RE, Howard J, Gutman JM, Gottesman M, Abou-Fayssal N, Wolintz R, Keilson M, Fernandez-Carbonell C, Krupp LB, Zhovtis Ryerson L (2020) COVID-19 outcomes in MS: Observational study of early experience from NYU Multiple Sclerosis Comprehensive Care Center. Neurol Neuroimmunol Neuroinflamm 7:e835

13. Singh S, Desai R, Gandhi Z, Fong HK, Doreswamy S, Desai V, Chockalingam A, Mehta PK, Sachdeva R, Kumar G (2020) Takotsubo syndrome in patients with COVID-19: a systematic review of published cases. SN Compr Clin Med 6:1-7

14. Valencia-Sanchez C, Goodman BP, Carter JL, Wingerchuk DM (2019) The spectrum of acute cardiopulmonary events associated with multiple sclerosis exacerbations. Mult Scler 25:758-765 